\title{
Continuous subcutaneous insulin infusion combined with liraglutide reduced glycemic variability and oxidative stress in type 2 diabetes mellitus: a study based on the flash glucose monitoring system
}

\author{
Li-qin $\mathrm{Li}^{1), 2)}$, Ming-yan $\mathrm{Yao}^{1), 2)}$, Jian-xia $\mathrm{Ma}^{1)}$, Peng Xue ${ }^{1)}$ and $\mathrm{Yu}-\mathrm{kun}^{\mathrm{Li}}{ }^{1)}$ \\ 1) Department of Endocrinology, The Third Hospital of Hebei Medical University, Shijiazhuang 050051, China \\ 2) Department of Endocrinology, Baoding No.1 Central Hospital, Baoding 071000, China
}

\begin{abstract}
We aimed to explore the use of the flash glucose monitoring (FGM) system in hospitalized newly diagnosed type 2 diabetes mellitus (T2DM) patients and to evaluate a new combination therapy of continuous subcutaneous insulin infusion (CSII) with or without liraglutide. This was an open-label, randomized study that was conducted in 60 newly diagnosed T2DM patients. The patients were randomized to receive either CSII $(n=30)$ or CSII + liraglutide $(n=30)$. The FGM system was used to assess the glycemic control and glycemic variability (GV) indices for 2 weeks. Mean blood glucose concentration (MBG), estimated hemoglobin A1c (HbA1c), and measures of GV, including the standard deviation of the mean glucose (SD), coefficient of variation (CV), interquartile range (IQR), mean amplitude of glycemic excursions (MAGE), largest amplitude of glycemic excursions (LAGE), and mean of daily difference (MODD) were compared between the two groups. Two oxidative stress biomarkers, 4-hydroxynonenal (4-HNE) and 8-hydroxydeoxyguanosine (8-OHdG), were measured before and after treatment. The estimated $\mathrm{HbA1c}$ and MBG decreased in both groups, especially the CSII + liraglutide group. SD, IQR, LAGE, and MODD were significantly lower in the CSII + liraglutide group than in the CSII group (all $p<0.05$ ); there was no difference in CV or MAGE $(p>0.05)$. Similarly, the 4-HNE and 8-OHdG levels were significantly lower in the CSII + liraglutide group $(p<0.05)$. Our findings suggest that CSII with liraglutide was superior to CSII monotherapy in improving glycemic control and glycemic variability and in decreasing oxidative stress markers. Flash glucose monitoring can successfully provide ambulatory glucose profile data in the real world.
\end{abstract}

Key words: Glycemic variability, Flash glucose monitoring system, Continuous subcutaneous insulin infusion, Liraglutide, Oxidative stress

GLUCOSE MONITORING is important for patients treated with insulin. Conventional glucose monitoring requires a blood sample, typically obtained by pricking the finger, a painful experience. A new sensor-based system called "flash glucose monitoring (FGM)" received Food and Drug Administration (FDA) approval in 2016. The new system painlessly monitors glucose levels with a sensor worn on the arm, without requiring blood samples. The sensor can sense blood glucose at 15-min intervals throughout the day and store the glucose data for up to 14 days. Worldwide, the majority of studies on FGM have focused on accuracy and safety, and it has proven safe and accurate [1-4] compared with conventional self-

Submitted Jan. 23, 2019; Accepted May 13, 2019 as EJ19-0016 Released online in J-STAGE as advance publication Jun. 27, 2019 Correspondence to: Yu-kun Li, Department of Endocrinology, The Third Hospital of Hebei Medical University, 139 Ziqiang Road, Shijiazhuang, 050051, China.

E-mail: yk119621225@163.com monitoring of blood glucose testing (SMBG) or continuous glucose monitoring systems (CGMs). The use of flash glucose monitoring was associated with a reduction in hypoglycemia in type 1 and type 2 diabetes mellitus patients and pregnant women. Furthermore, studies have indicated high patient acceptability and satisfaction with the system [5-7]. Few studies have assessed the clinical use and experience of this new technology in newly diagnosed type 2 diabetes patients who need continuous subcutaneous insulin infusion (CSII) with or without other treatments. Therefore, we aimed to investigate the use of flash glucose monitoring in real clinical practice.

Many studies have shown that CSII can benefit newly diagnosed, poorly controlled type 2 diabetes mellitus (T2DM) [8]. CSII was recommended for selected patients with newly diagnosed T2DM in the Chinese guidelines [9]. Liraglutide, a long-acting glucagon-like peptide-1 (GLP-1) analog, has been reported to improve glycemic control with a low risk of hypoglycemia, 
increase insulin sensitivity, protect $\beta$-cell function and reduce glycemic variability (GV) [10]. CSII with liraglutide is a new combination therapy, specifically for newly diagnosed type 2 diabetes. We hypothesized that this combination therapy would further improve glycemic control or GV, with a marked diminishment in oxidative stress markers. FGM can be employed to detect glucose fluctuations that are impossible to identify with SMBG. In this study on the FGM system, participants were assigned to receive CSII alone or with liraglutide. We aimed to investigate whether the addition of liraglutide to CSII would reduce GV, as assessed by ambulatory glucose profile (APG) data, or lead to reduced oxidative stress as shown by lower 4-hydroxynonenal (4-HNE) and 8-hydroxydeoxyguanosine $(8-\mathrm{OHdG})$ levels. This is the first clinical use of a flash continuous glucose monitoring system to investigate glycemic control and glycemic variability in Chinese patients with newly diagnosed T2DM with a poor glucose state who needed to receive CSII alone or in combination with liraglutide. Our goal was to find a perfect combination of FGM, CSII, and liraglutide for uncontrolled, newly diagnosed T2DM patients who need intensive insulin treatment in the hospital.

\section{Materials and Methods}

\section{Patients}

A total of 68 newly diagnosed T2DM patients were enrolled in this prospective study. This study was approved by the Third Hospital of Hebei Medical University in China and was conducted in accordance with the Declaration of Helsinki. Ethics - informed consent was obtained from all patients. All patients were willing to use a flash glucose monitoring system. Of the 68 patients, 60 finished the treatment. This study was accomplished in 2 weeks.

Inclusion criteria were newly diagnosed T2DM defined by the American Diabetes Association (ADA), age 25-70 years, hemoglobin A1c (HbA1c) 9-14\%, and body mass index (BMI) $25-35 \mathrm{~kg} / \mathrm{m}^{2}$.

Exclusion criteria included type 1 diabetes or diabetes with identified secondary causes, diabetic ketoacidosis, active coronary artery disease, severe liver or kidney diseases, infectious or gastrointestinal diseases, newly diagnosed cancer, pregnancy or breast-feeding while receiving GLP-1 receptor agonists or DPP-4 inhibitors or with any contraindication to liraglutide, and other reasons causing a patient to be unsuitable for the study.

\section{Study design}

The study was carried out in 68 patients with type 2 diabetes who were admitted to the hospital of Hebei
Medical University in China for glycemic control. Five did not meet the inclusion criteria, 3 failed to accept the treatment, and 60 completed the study. Every patient provided a full medical and medication history and underwent an electrocardiogram and a physical examination (including body weight, height, and blood pressure) at study enrollment. Fasting venous blood samples were obtained for laboratory assessment. All patients were given an education program on diabetes self-management, including diet and exercise counseling, at baseline. A diabetic diet consisting of $50 \%$ carbohydrate $(200 \mathrm{~g})$, $35 \%$ fat, and $15 \%$ protein was provided for all subjects during the intervention. The distribution of caloric intake was $20 \%$ for breakfast, $40 \%$ for lunch, and $40 \%$ for dinner. Regular physical exercise, such as walking, jogging, or stair climbing, for $30 \mathrm{~min}$ after meals was recommended. Patients were provided with the flash glucose monitoring system (Freestyle Libre) at baseline and were asked to apply it for two weeks throughout the treatment. According to computer-generated random orders, the patients were randomized 1:1 to either the CSII alone group (CSII alone group) or CSII combined with once-daily liraglutide group (CSII + liraglutide) for 2 weeks. Patients and investigators were not blinded. Patients in both groups were given insulin aspart (NovoRapid, Novo Nordisk, Bagsværd, Denmark) with an insulin pump (MiniMed 712, Medtronic, Northridge, CA), whereas the CSII + liraglutide group was given an additional liraglutide injection (Victoza, Novo Nordisk, Bagsvaerd, Denmark). The initial insulin dosage was $0.5-0.7 \mathrm{IU} / \mathrm{kg} / \mathrm{day}$, with the total daily dosage divided $50 / 50$ into basal and bolus infusions. The initial basal dose was divided into 24 doses. The basal and bolus doses of insulin infusion were adjusted daily by one doctor by 2 to 10 units according to the blood glucose level to achieve euglycemia (fasting blood glucose $<7.0$ $\mathrm{mmol} / \mathrm{L}$ and postprandial blood glucose $<10.0 \mathrm{mmol} / \mathrm{L}$ ). Liraglutide was injected at 7 am every day and began at $0.6 \mathrm{mg} / \mathrm{day}$; it was increased every 3 days from the initial dose of $0.6 \mathrm{mg} /$ day to $1.2 \mathrm{mg} /$ day up to $1.8 \mathrm{mg}$ /day if there was no intolerance, such as vomiting or nausea. The sensors were erased after 2 weeks, and the glucose data were downloaded from the flash glucose monitoring systems.

\section{Sensors and readers}

All participants wore a FreeStyle Libre device, which is a sensor-based glucose monitor. A small sensor was inserted easily, speedily, and painlessly on the back of the upper arm, which pushes a small, flexible filament into the skin and stays there for 14 days. The reader can be waved over the sensor to see real-time glucose values as often as the patient wishes. However, for a complete 
glycemic picture, the participant should scan it at least once every 8 hours, and 7 times per day is better (before and $2 \mathrm{~h}$ after each meal and at bedtime). The dedicated reader provides glucose values and a trend of glucose, and the reader also shows the past 8 hours of data, which are automatically stored every $15 \mathrm{~min}$. It holds 90 days of data. After completion of the FGM intervention, the glucose data were downloaded. The following glycemic variability indices obtained from the FGM were calculated using E-Followup software from Zion China [11]: mean amplitude of glycemic excursion (MAGE), mean of daily difference (MODD), standard deviation (SD), coefficient of variation (CV), interquartile range (IQR) and largest amplitude of glycemic excursions (LAGEs).

\section{Biochemical assays}

Plasma glucose, hemoglobin A1c (HbA1c), fasting Cpeptide, insulin, total cholesterol, LDL-cholesterol, HDL cholesterol, triglycerides, and renal and liver function were measured in the laboratory of the Third Hospital of Hebei Medical University at baseline. HbAlc was assayed using high-performance liquid chromatography and is expressed as a National Glycohemoglobin Standardization Program unit. Other blood samples were centrifuged $\left(1,500 \times \mathrm{g}\right.$ for $15 \mathrm{~min}$ at $\left.4^{\circ} \mathrm{C}\right)$, and the supernatant plasma was stored at $-80^{\circ} \mathrm{C}$ until use.

4-Hydroxynonenal (4-HNE), 8-hydroxydeoxyguanosine (8-OHdG) and 1,5-anhydroglucitol (1,5-AG) were measured at baseline and 2 weeks after treatment. 4-HNE was measured by a Human 4-Hydroxynonenal (HNE) ELISA Kit (Catalog Number. CSB-E16214h, CUSABIO), with a sensitivity of $0.5 \mathrm{pg} / \mathrm{mL}$ and a range of $39-2,500 \mathrm{pg} / \mathrm{mL}$. The intra-assay variation was less than $8.0 \%$, and the interassay variation was less than $10 \%$. $8-\mathrm{OHdG}$ was measured by a Human 8-Hydroxydeoxyguanosine (8OHdG) ELISA Kit (Catalog Number, CSB-E10140h, CUSABIO) with a sensitivity of $0.8 \mathrm{ng} / \mathrm{mL}$ and a range of $2-800 \mathrm{ng} / \mathrm{mL}$. The intra-assay variation was less than $6.0 \%$, and the interassay variation was less than $11 \%$. Serum 1,5-anhydroglucitol (1,5-AG) levels were measured by a 1,5-Anhydroglucitol Enzyme-linked Immunosorbent Assay Kit (CEB046Ge, CLOUD-CLONE CORP.), with a sensitivity of $0.7 \mu \mathrm{g} / \mathrm{mL}$ and a range of $1.9-150 \mu \mathrm{g} / \mathrm{mL}$. The intra-assay variation was less than $10 \%$, and the interassay variation was less than $12 \%$.

\section{Statistical analysis}

The primary endpoints of our study were the betweengroup differences in GV (including SD, CV, IQR, and MAGE, LAGE, MODD). Secondary outcomes included all other variables regarding between-group differences in MBG and estimated $\mathrm{HbAlc}$, hypoglycemia rate (defined as blood glucose $\leq 3.9 \mathrm{mmol} / \mathrm{L}$ ), in-target rate (defined as blood glucose between 3.9-10.0 mmol/L), and hyperglycemia rate (defined as blood glucose $\geq 10.0$ $\mathrm{mmol} / \mathrm{L}$ ). The changes in insulin dosage, body weight, and plasma 4-HNE, and 8-OHdG levels were also included as secondary outcomes. The sample size of each primary endpoint was calculated by the standard formula for a two-sided, two-sample $t$ test using PASS 11.0 software. Considering a dropout rate of $20 \%$, a total of 68 patients were recruited, and 60 patients completed the trial and were included in the analysis. The sample size would provide more than $80 \%$ power at $5 \%$ significance.

All analyses were carried out using the Statistical Package for Social Sciences (SPSS) version 22.0 (SPSS, Chicago, IL, USA). Data are reported as mean and standard deviation for normally distributed continuous variables and median with the interquartile range for the nonnormally distributed continuous variables. Comparisons between the groups were made using Student's $t$-test and Wilcoxon's rank test for normally and nonnormally distributed continuous variables, respectively. Paired-sample $T$-tests or Wilcoxon signed rank test were performed to estimate the changes from before to after the intervention. The $\chi^{2}$ test was applied to analyze the differences in proportions. A $p$-value of less than 0.05 was considered statistically significant.

\section{Results}

\section{Baseline characteristics of the two groups}

No severe hypoglycemia occurred throughout the therapy. There were no between-group differences in age, sex and BMI at baseline. Additionally, there were no significant differences between the two groups in glucose levels (HbA1c, FPG), blood pressure, lipid profile (TC, HDL-C, LDL-C, and TG), C-peptide, fasting insulin, diabetic complications, or family history at the beginning of the study (all $p>0.05$ ). The clinical characteristics and biochemical variables for the two groups are summarized in Table 1.

\section{The glucose data and glycemic variability of the two different groups}

The percentage of time spent within 3.9-10.0 mmol/L (in-target rate) was significantly higher, while the percentage of time spent above $10.0 \mathrm{mmol} / \mathrm{L}, 13.9 \mathrm{mmol} / \mathrm{L}$, or $22.2 \mathrm{mmol} / \mathrm{L}$, also called the hyperglycemia rate, was lower, in the CSII + liraglutide group. There was no difference in time spent below $2.8 \mathrm{mmol} / \mathrm{L}, 3.3 \mathrm{mmol} / \mathrm{L}$, or $3.9 \mathrm{mmol} / \mathrm{L}$, also known as the hypoglycemia rate, between the two groups (all $p>0.05$, Fig. 1). The CSII + liraglutide group showed a significantly lower estimated MBG and HbA1c than the CSII alone group (all $p<$ 
Table 1 Patients' characteristics at baseline

\begin{tabular}{|c|c|c|c|}
\hline Items & CSII group & CSII + Liraglutide & $p$ \\
\hline Number & 30 & 30 & - \\
\hline Gender (Female/Male) & $3 / 27$ & $7 / 23$ & 0.166 \\
\hline Age (year) & $46.00(42.50,59.00)$ & $50.00(42.50,66.00)$ & 0.615 \\
\hline SBP (mmHg) & $139.00 \pm 17.08$ & $136.27 \pm 18.63$ & 0.556 \\
\hline DBP (mmHg) & $94.10 \pm 11.77$ & $94.30 \pm 13.66$ & 0.952 \\
\hline BMI $\left(\mathrm{kg} / \mathrm{m}^{2}\right)$ & $29.74(26.33,29.98)$ & $27.30(25.90,28.72)$ & 0.111 \\
\hline $\mathrm{HbA1c}(\%)$ & $11.20(9.30,12.80)$ & $10.40(10.30,11.80)$ & 0.855 \\
\hline $\mathrm{FBG}(\mathrm{mmol} / \mathrm{L})$ & $12.97 \pm 1.90$ & $13.50 \pm 2.14$ & 0.379 \\
\hline FC-P (ng/mL) & $2.05 \pm 1.38$ & $2.04 \pm 1.23$ & 0.972 \\
\hline Fasting insulin $(\mu \mathrm{U} / \mathrm{mL})$ & $12.30(7.98,16.00)$ & $12.09(7.98,15.09)$ & 0.870 \\
\hline $\operatorname{Tg}(\mathrm{mmol} / \mathrm{L})$ & $2.07(1.16,4.24)$ & $1.64(1.11,4.72)$ & 0.756 \\
\hline $\mathrm{TC}(\mathrm{mmol} / \mathrm{L})$ & $5.07 \pm 1.60$ & $4.92 \pm 1.81$ & 0.734 \\
\hline LDL-c (mmol/L) & $2.97 \pm 1.07$ & $2.87 \pm 1.24$ & 0.564 \\
\hline HDL-c (mmol/L) & $1.21(1.11,1.40)$ & $1.21(1.12,1.43)$ & 0.564 \\
\hline CRP (mg/L) & $1.92(1.14,5.47)$ & $1.92(0.53,8.29)$ & 1.000 \\
\hline UA (ummol/L) & $364.00(279.00,421.50)$ & $346.00(270.00,430.00)$ & 0.505 \\
\hline Family history of T2DM (\%) & 50.0 & 46.67 & 0.796 \\
\hline Peripheral vascular disease (\%) & 26.67 & 40.00 & 0.273 \\
\hline Peripheral neuropathy (\%) & 23.33 & 13.33 & 0.317 \\
\hline Retinopathy (\%) & 6.66 & 16.67 & 0.421 \\
\hline Microalbuminuria (\%) & 16.67 & 13.33 & 0.718 \\
\hline
\end{tabular}

Note: data are presented as the means (SD) or median (Q1-Q3). SBP, systolic blood pressure; DBP, diastolic blood pressure; BMI, body mass index; HbAlc, glycated hemoglobin; CSII group, CSII alone group; CSII + liraglutide group, CSII therapy in combination with liraglutide group; FBG, fasting plasma glucose; FC-P, fasting C-peptide; TC, total cholesterol; Tg, triglycerides; HDL-C, highdensity lipoprotein cholesterol; LDL-C, low-density lipoprotein cholesterol. UA, uric acid.

0.05, Fig. 1). After 14 days of treatment, the SD, CV, IQR, MAGE, LAGE, and MODD all decreased in both groups, but the SD, IQR, LAGE, and MODD parameters were significantly lower in the liraglutide + CSII group than in the CSII alone group (all $p<0.05$, Fig. 2), There was no significant difference between the two groups in CV or MAGE ( $p>0.05$, Fig. 2).

\section{Levels of oxidative stress factors and serum 1,5-AG}

The oxidative stress markers 8-OHdG and 4-HNE levels were comparable between the two groups at baseline (all $p>0.05$ ). The 8-OHdG and 4-HNE levels were significantly decreased in both groups after treatment (both $p<0.05$, Table 2). The decreases in 8-OHdG and 4-HNE levels in the CSII + liraglutide group were significantly greater than the reductions in the CSII group ( $p=0.044$ and $p=0.013$, respectively, Table 2).

Serum 1,5-AG levels were similar between the two groups at baseline, and both levels increased after treat- ment. Serum 1,5-AG levels in the CSII combined with liraglutide group were significantly higher than those in the CSII alone group ( $p<0.05$, Table 2$)$.

\section{Changes in body weight and insulin dose during the study}

Body weight loss was significantly greater in the CSII + liraglutide group than in the CSII group $(-0.47 \pm 0.65$ vs. $-1.25 \pm 0.57, p<0.05)$. As shown in Table 3 , the daily insulin dosage in the CSII + liraglutide group $(0.20$ $\pm 0.12 \mathrm{IU} / \mathrm{kg} /$ day) was significantly lower than that in the CSII group $(0.43 \pm 0.12 \mathrm{IU} / \mathrm{kg} /$ day; $p<0.05)$. The decline in the daily insulin dosage was significantly greater in the CSII + liraglutide group than in the CSII therapy group $(0.34 \pm 0.12 v s .0 .16 \pm 0.15, p<0.05)$.

\section{Safety}

Gastrointestinal disorders such as nausea, vomiting, and diarrhea happened in the liraglutide group. One epi- 

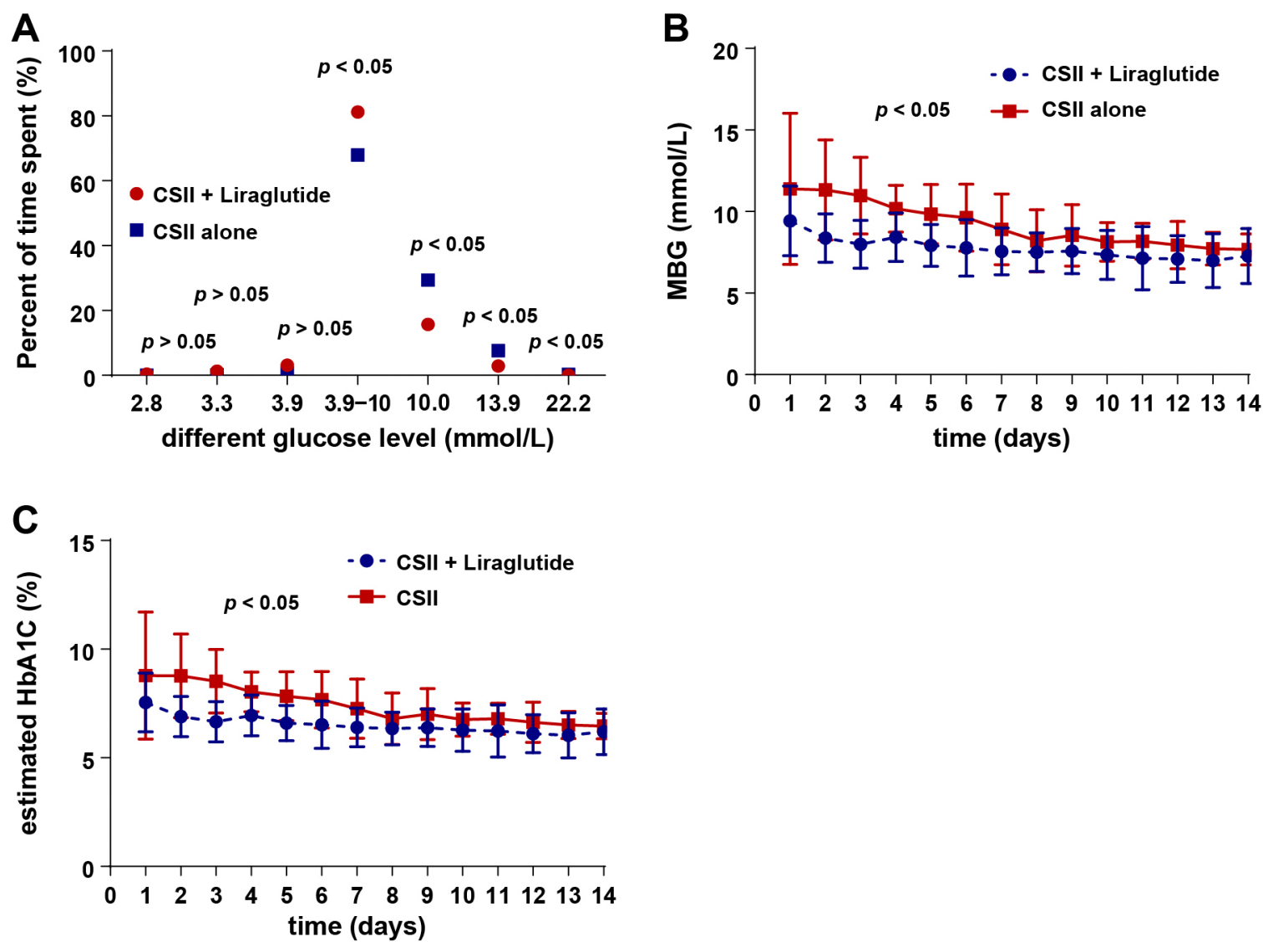

Fig. 1 Glucose data derived from the FGM after 14 days of treatment.

The percentage of time spent within $3.9-10.0 \mathrm{mmol} / \mathrm{L}$ was significantly higher, while the percentage of time spent above 10.0 $\mathrm{mmol} / \mathrm{L}, 13.9 \mathrm{mmol} / \mathrm{L}$, or $22.2 \mathrm{mmol} / \mathrm{L}$ was significantly lower, in the CSII + liraglutide group $(p<0.05)$. There was no difference in time spent below $2.8 \mathrm{mmol} / \mathrm{L}, 3.3 \mathrm{mmol} / \mathrm{L}$, or $3.9 \mathrm{mmol} / \mathrm{L}$ between the two groups $(p>0.05)$ (Fig. 1A). The CSII combined with liraglutide group showed a lower MBG and HbA1c than the control group $(p<0.05)$ (Fig. 1B, Fig. 1C).

Note: PT 2.8, percentage of time spent below $2.8 \mathrm{mmol} / \mathrm{L}$; PT 3.3, percentage of time spent below $3.3 \mathrm{mmol} / \mathrm{L}$; PT 3.9, percentage of time spent below $3.9 \mathrm{mmol} / \mathrm{L}$; PT 3.9-10.0, percentage of time spent between 3.9 and $10.0 \mathrm{mmol} / \mathrm{L}$; PT 10, percentage of time spent above $10.0 \mathrm{mmol} / \mathrm{L}$; PT 13.9, percentage of time spent above $13.9 \mathrm{mmol} / \mathrm{L}$; PT 22.2, percentage of time spent above 22.2 mmol/L; estimated HbAlc, estimated hemoglobin A1c; MBG, mean blood glucose concentration; CSII, continuous subcutaneous insulin infusion; CSII + liraglutide, continuous subcutaneous insulin infusion combined with liraglutide.

sode or more of nausea was experienced by $16.7 \%$, vomiting was experienced by $10 \%$, and diarrhea was experienced by $26.7 \%$. The gastrointestinal disorders were generally mild and transient. In fact, 29 patients could tolerate the maximum dose of liraglutide, but because one patient could not tolerate $1.8 \mathrm{mg}$ of liraglutide, we reverted to $1.2 \mathrm{mg}$. Overall, liraglutide was well tolerated, and no patients withdrew because of gastrointestinal disorders. During the therapy, the incidence of hypoglycemia, which was defined as a blood glucose level $<3.9 \mathrm{mmol} / \mathrm{L}$, was similar between the CSII alone group and the CSII + liraglutide group ( $p=0.200$, Table $3)$. Most of the hypoglycemic episodes were mild and could be corrected. No severe hypoglycemia was recorded in either group.

\section{Discussion}

The Flash Glucose Monitoring System has come into use in recent years and is useful for monitoring daily glucose and detecting hypoglycemic events [12]. Glycemic variability has been proven to activate serious inflammatory reactions and oxidative stress, which could play a key role in inducing the complications of diabetes. Controlling glycemic variability seems to better prevent diabetic complications [13-15]. Most studies on glucose variability have been based on CGMS, and to the best of our knowledge, there is no published clinical trial based on the FGM system to explore the effects of liraglutide add-on therapy in newly diagnosed type 2 diabetes. This study used FGM to directly compare the effect of CSII with or without liraglutide on GV and oxidative stress. The major findings of the present study are summarized 

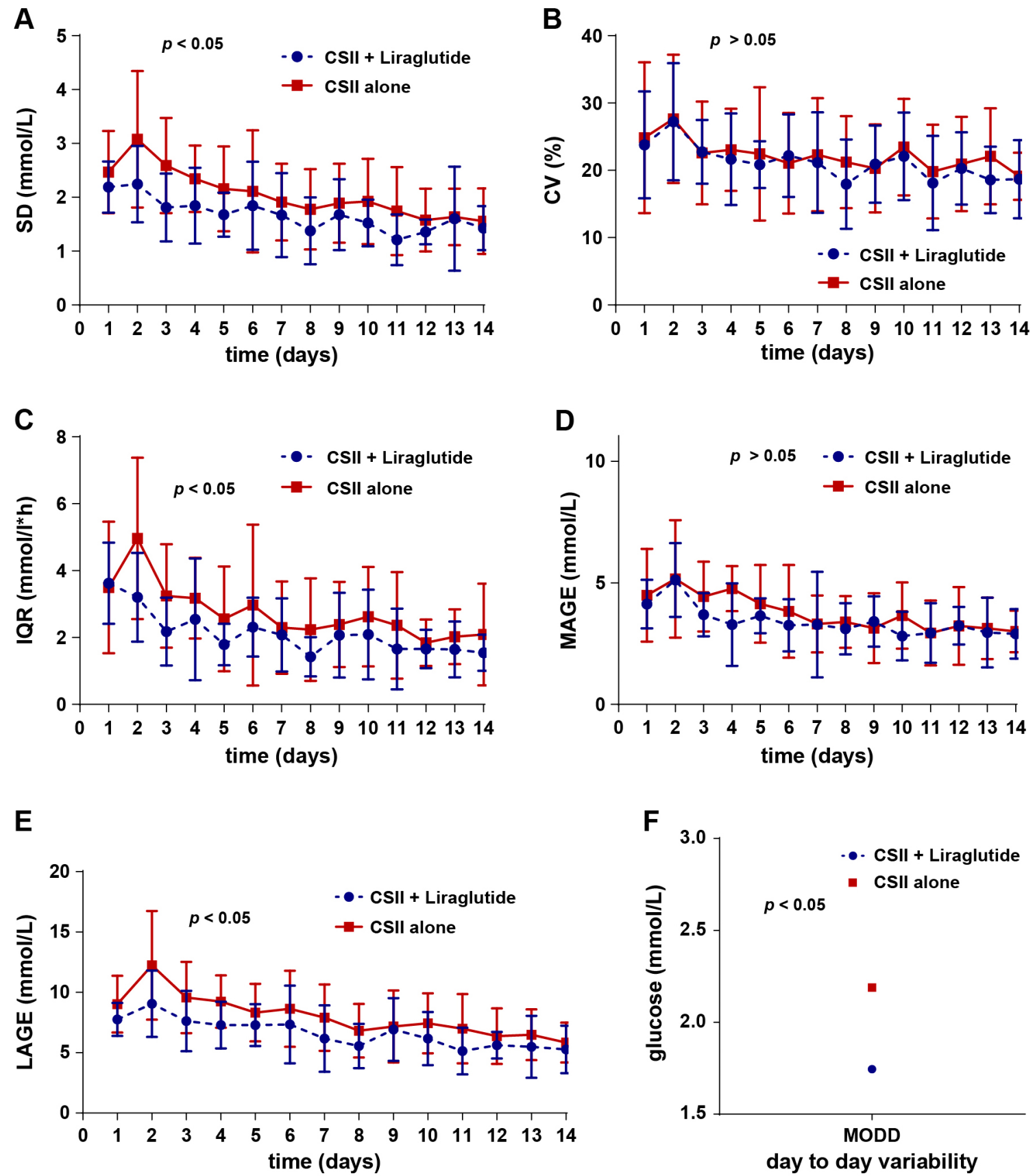

Fig. 2 The 14-day glycemic variability parameters assessed by the FGM system.

The glycemic variability parameters SD, IQR, LAGE, and MODD were significantly decreased in the CSII with liraglutide group compared with the CSII alone group. There was no significant difference between the two groups in CV or MAGE.

Note: SD, standard deviation; CV, coefficient of variation; IQR, interquartile range; LAGE, large amplitude of glycemic excursion; MAGE, mean amplitude of glycemic excursion; MODD, mean daily difference.

as follows. First, glycemic parameters derived from the FGM system, such as the SD, IQR, LAGE, and MODD, were significantly lower in the CSII + liraglutide group, suggesting that the addition of liraglutide narrowed the $\mathrm{GV}$, which is similar to previous studies based on CGMS. Second, we measured some markers of oxidative stress in our study. The levels of 4-HNE and 8-OHdG were lower in the CSII combined with liraglutide group than in the CSII alone group, which indicates that the addition of liraglutide may reduce oxidative stress more than CSII alone. CSII-based short-term intensive treatment in patients with newly diagnosed type 2 diabetes is very effective in improving glycemic and lipid control, $\beta$-cell function, and insulin sensitivity, and it is thought to be suitable for newly diagnosed type 2 diabetes, especially for patients with $\mathrm{HbA1c}>9 \%$ [16]. Recent studies 
Table 2 8-OHdG, 4-HNE, and 1,5-AG levels in the different groups

\begin{tabular}{lcccc}
\hline Items & & CSII + Liraglutide & CSII & $p$ \\
\hline & baseline & $352.35(314.08,430.72)$ & $364.09(274.05,850.89)$ & 0.751 \\
8-OHdG $(\mathrm{ng} / \mathrm{mL})$ & after & $167.53(131.08,223.80)$ & $237.06(164.00,439.25)$ & 0.011 \\
& P-after/baseline & 0.000 & 0.000 & - \\
& Change & $-193.47(-232.51,-159.74)$ & $-154.69(-209.25,-64.87)$ & 0.044 \\
\hline & baseline & $50,108.25(39,534.91,63,501.10)$ & $51,497.7(33,868.18,81,113.15)$ & 0.647 \\
$4-\mathrm{HNE}(\mathrm{pg} / \mathrm{mL})$ & after & $30,590.22(22,389.22,41,759.50)$ & $37,958.1(30,301.97,62,038.87)$ & 0.014 \\
& P-after/baseline & 0.000 & 0.003 & - \\
\hline & Change & $-17,145.6(-31,331.84,-12,073.31)$ & $-10,484(-27,457.84,-4,047.96)$ & 0.013 \\
\hline & baseline & $6.16(1.53)$ & $6.26(2.05)$ & 0.826 \\
\hline 1,5-AG $(\mathrm{ug} / \mathrm{mL})$ & after & $14.24(3.24)$ & $12.23(3.7)$ & 0.029 \\
& P-after/baseline & 0.000 & 0.000 & - \\
\hline
\end{tabular}

Note: 8-OHdG, 8-hydroxydeoxyguanosine; 4-HNE, 4-hydroxynonenal; 1,5-AG, 1,5-anhydroglucitol

Table 3 Patients' characteristics after treatment

\begin{tabular}{lccc}
\hline Items & CSII & CSII + liraglutide & $p$ \\
\hline Incidence of hypoglycemia (\%) & $0.30(0.2,0.8)$ & $0.50(0.20,3.00)$ & 0.200 \\
Change of body weight (kg) & $-0.47 \pm 0.65$ & $-1.25 \pm 0.57$ & 0.000 \\
\hline Final daily insulin dosage (IU/kg/day) & $0.43 \pm 0.12$ & $0.20 \pm 0.12$ & 0.000 \\
\hline Change of insulin dosage (IU/kg/day) & $0.16 \pm 0.15$ & $0.34 \pm 0.12$ & 0.000 \\
\hline Nausea (\%) & - & $16.7 \%$ & - \\
\hline Diarrhea (\%) & - & $10.0 \%$ & - \\
Vomiting (\%) & - & $26.7 \%$ & - \\
\hline Gastrointestinal symptoms (\%) & - & $53.3 \%$ & - \\
\hline $1.2 / 1.8$ mg liraglutide & - & $1 / 29$ & - \\
\hline
\end{tabular}

have reported that liraglutide can potentially reduce the risk of cardiovascular disease [17], improve $\beta$-cell function, reduce GV $[18,19]$, and decrease oxidative stress [20]. Several studies have proven that the addition of liraglutide is safe and effective in patients with inadequately controlled T2DM $[21,22]$. However, the combination of liraglutide and CSII in newly diagnosed type 2 diabetes remains inconclusive. In this study, we compared the differences in the glucose data and oxidative stress after intervention with or without the combination of liraglutide. With the flash glucose monitoring technology system, we could easily obtain an integrated picture of glucose data and glucose variability with no pain. Glycemic data (Fig. 1) showed that the addition of liraglutide improved glycemic control compared with CSII alone, manifested by decreased FBG and estimated HbA1c. As previous studies have shown, 1,5-AG is a new clinical marker for glycemic control in patients with type 2 diabetes $[23,24]$. In this study, we found that the serum 1,5-AG concentration in the CSII combined with liraglutide group was significantly higher than that in the CSII alone group (Table 2), which may further imply that glycemic control with CSII combined with liraglutide is better than with CSII. In addition, our analysis of GV (Fig. 2) revealed that the use of liraglutide in combination with CSII reduced blood glycemic variability. Not only did the SD, IQR, and LAGE, which reflect the intraday glycemic variability, improve, but the MODD, which represents the day-to-day glycemic variability, decreased. A clinical study on another GLP-1 analog, exenatide [25], also demonstrated that the addition of exenatide to CSII decreased glucose variability, exhibited by a significant reduction in MAGE. Our findings are in accordance with this previous study and corroborate the finding that the addition of GLP-1 analog to CSII in poorly controlled T2DM can improve GV. W. Lane [10] 
reported a similar result, in which 37 type 2 diabetes patients who received intensive insulin treatment (continuous subcutaneous insulin infusion or multiple daily injections) were assigned to combined treatment with or without liraglutide. The addition of liraglutide led to a greater reduction in $\mathrm{HbA} 1 \mathrm{c}$ and narrowed glycemic variability, which was manifested by reduced average daily standard deviation (SD) in that study. Similar findings [26] also appeared in a well-designed study in newly diagnosed type 2 diabetes patients who were treated by CSII with or without liraglutide. Liraglutide combined with CSII improved glycemic control and $\beta$-cell function, which is consistent with our findings, though that pilot study also observed that the improved $\beta$-cell function faded away after stopping the liraglutide. No serious hypoglycemic events happened during this study, and it is possible that the FGM system itself contributed to our result. This finding confirms the safety of the FGM system in clinical use. These findings indicate the therapeutic advantage of the addition of liraglutide to CSII treatment in newly diagnosed type 2 patients, and they support the concept that CSII with liraglutide treatment can successfully manage and benefit newly diagnosed T2DM.

A relationship between diabetes and oxidative stress has been observed [27]. Recently, there has been increasing interest in the antioxidant effect of therapy for type 2 diabetes [28]. Liraglutide has been demonstrated to have antioxidative and anti-inflammatory effects [29, 30]. 8Hydroxy deoxyguanine (8-OHdG), a DNA-damaging oxidative stress biomarker, could be seen as a promising biomarker in T2DM. T2DM patients have shown significantly higher levels of serum 8-OHdG compared to control groups. This biomarker is beneficial for early diagnosis, effective therapeutic strategies and monitoring in T2DM [31-34]. 4-Hydroxynonenal (4-HNE) is a major biomarker of oxidative stress and lipid peroxidation, and it is thought that 4-HNE-induced oxidative stress plays a role in the regulation of preadipocyte growth, differentiation and insulin signaling, which may contribute to adipose tissue metabolic dysfunction associated with insulin resistance $[35,36]$. Our study used 8OHdG and 4-HNE activity to assess the oxidative activity of the two groups. Our blood sample analysis suggested that CSII + liraglutide significantly decreased the 4-HNE and 8-OHdG levels compared with CSII alone. These results confirm that the addition of liraglutide can decrease oxidative stress, in line with a previous study [30]. The addition of liraglutide to metformin resulted in improvement in oxidative stress as well as plasma ghrelin and HO-1 concentrations in T2DM patients. These data support our hypothesis that liraglutide further improves oxidative stress. As other studies have indicated, in addition to glycemic control, oxidative stress can also contribute to the development of T2DMrelated cardiovascular disease and diabetic complications. Improvement in oxidative stress may contribute to the improvement in cardiovascular diseases and diabetic complications.

Altogether, our findings are in accordance with previous studies and substantiate the findings that the addition of liraglutide to CSII in newly diagnosed, poorly controlled type 2 diabetes not only improves the MBG but also reduces the $\mathrm{GV}$ and oxidative stress.

Limits: Although these results clearly demonstrate the effectiveness of liraglutide add-on therapy, our study has several limitations. First, the number of patients was relatively small, and the duration of the study was relatively short. The monitoring period was only 14 days. It is better to prolong the treatment to 3 months, 6 months, or 1 year, so longer studies with larger sample sizes are needed. Further studies are needed to examine the efficacy of long-term combination therapy in a larger cohort of subjects with type 2 diabetes mellitus. Second, as the FGM system needed no calibration, the participants were instructed to test their capillary blood glucose only on the first day, and if symptoms of hypo- or hyperglycemia occurred, if the sensor failed or if the sensed glucose was out of the device's range. We did not use other methods of measuring blood glucose at the same time, such as CGMS or SMBG every day. However, a previous study has demonstrated the accuracy of the FGM system. Third, subjects enrolled in this study had a poor glucose state, so the effects of treatment in subjects with HbA1c of less than $9 \%$ remain unknown. Thus, to confirm the usefulness of the FGM system for T2DM, we need further evaluation in a large-scale study over a longer period. Further studies may be required to explore whether CSII with or without treatment will have different influences on the following treatment after the study.

\section{Conclusions}

The FGM system can provide ideal glucose data for glycemic state and glycemic variability. In this study, we confirmed that the FGM system appears to be clinically safe and useful in the clinical treatment of hospitalized patients. Liraglutide add-on therapy to CSII not only led to improved glucose control but also reduced the GV and oxidative stress in poorly controlled newly diagnosed type 2 diabetes. The results of this study suggest that the combination therapy of liraglutide and CSII is a potential therapeutic option for newly diagnosed T2DM. To verify this hypothesis, we need a further prospective study with a larger number of patients over a longer period of time. 


\section{References}

1. Haak T, Hanaire H, Ajjan R, Hermanns N, Riveline JP, et al. (2017) Flash glucose-sensing technology as a replacement for blood glucose monitoring for the management of insulin-treated type 2 diabetes: a multicenter, open-label randomized controlled trial. Diabetes Ther 8: 55-73.

2. Bonora B, Maran A, Ciciliot S, Avogaro A, Fadini GP (2016) Head-to-head comparison between flash and continuous glucose monitoring systems in outpatients with type 1 diabetes. J Endocrinol Invest 39: 1391-1399.

3. Edge J, Acerini C, Campbell F, Hamilton-Shield J, Moudiotis C, et al. (2017) An alternative sensor-based method for glucose monitoring in children and young people with diabetes. Arch Dis Child 102: 543-549.

4. Scott EM, Bilous RW, Kautzky-Willer A (2018) Accuracy, user acceptability, and safety evaluation for the FreeStyle Libre Flash Glucose Monitoring System when used by pregnant women with diabetes. Diabetes Technol Ther 20: 180-188.

5. Bailey T, Bode BW, Christiansen MP, Klaff LJ, Alva S (2015) The performance and usability of a factorycalibrated flash glucose monitoring system. Diabetes Technol Ther 17: 787-794.

6. Bolinder J, Antuna R, Geelhoed-Duijvestijn P, Kröger J, Weitgasser R (2016) Novel glucose-sensing technology and hypoglycaemia in type 1 diabetes: a multicentre, nonmasked, randomised controlled trial. Lancet 388: 2254 2263.

7. Ólafsdóttir AF, Attvall S, Sandgren U, Dahlqvist S, Pivodic A, et al. (2016) A clinical trial of the accuracy and treatment experience of the flash glucose monitor freestyle libre in adults with type 1 diabetes. Diabetes Technol Ther 19: 164-172.

8. Chen A, Huang Z, Wan X, Deng W, Wu J, et al. (2012) Attitudes toward diabetes affects maintenance of drug-free remission in patients with newly diagnosed type 2 diabetes mellitus after short-term continuous subcutaneous insulin infusion treatment. Diabetes Care 35: 474-481.

9. Yang W, Weng J (2014) Early therapy for type 2 diabetes in China. Lancet Diabetes Endocrinol 2: 992-1002.

10. Lane W, Weinrib S, Rappaport J, Hale C (2014) The effect of addition of liraglutide to high-dose intensive insulin therapy: a randomized prospective trial. Diabetes Obes Metab 16: 827-832.

11. Ara A, Ara A (2017) Case study: integrating IoT, streaming analytics and machine learning to improve intelligent diabetes management system. International Conference on Energy, Communication, Data Analytics and Soft Computing (ICECDS): 3179-3182.

12. Leelarathna L, Wilmot EG (2018) Flash forward: a review of flash glucose monitoring. Diabet Med 35: 472-482.

13. Saisho Y (2014) Glycemic variability and oxidative stress: a link between diabetes and cardiovascular disease? Int $J$ Mol Sci 15: 18381-18406.

14. Brownlee M, Hirsch IB (2006) Glycemic variability: a hemoglobin A1c-independent risk factor for diabetic complications. JAMA 295: 1707-1708.

15. Prázný M, Škrha J, Šoupal J, Škrha J Jr (2017) Glycemic variability and microvascular complications of diabetes. Cas Lek Cesk 156: 308-313 (In Czech).

16. Weng J (2017) Short-term intensive insulin therapy could be the preferred option for new onset type 2 diabetes mellitus patients with $\mathrm{HbA1c}>9$. J Diabetes 9: 890-893.

17. Arturi F, Succurro E, Miceli S, Cloro C, Ruffo M, et al. (2017) Liraglutide improves cardiac function in patients with type 2 diabetes and chronic heart failure. Endocrine 57: 464-473.

18. Retnakaran R, Kramer CK, Choi H, Swaminathan B, Zinman B, et al. (2014) Liraglutide and the preservation of pancreatic $\beta$-cell function in early type 2 diabetes: the LIBRA trial. Diabetes Care 37: 3270-3278.

19. Lin CH, Hsieh SH, Sun JH, Tsai JS, Huang YY (2015) Glucose variability and $\beta$-cell response by GLP-1 analogue added-on CSII for patients with poorly controlled type 2 diabetes. Sci Rep 5: 16968.

20. Rizzo M, Abate N, Chandalia M, Rizvi AA, Giglio RV, et al. (2015) Liraglutide reduces oxidative stress and restores heme oxygenase- 1 and ghrelin levels in patients with type 2 diabetes: a prospective pilot study. J Clin Endocrinol Metab 100: 603-606.

21. Seino Y, Kaneko S, Fukuda S, Osonoi T, Shiraiwa T, et al. (2016) Combination therapy with liraglutide and insulin in Japanese patients with type 2 diabetes: a 36-week, randomized, double-blind, parallel-group trial. J Diabetes Investig 7: 565-573.

22. Kaneko S, Nishijima K, Bosch-Traberg H, Kaku K, Seino Y (2018) Efficacy and safety of adding liraglutide to existing insulin regimens in Japanese patients with type 2 diabetes mellitus: a post-hoc analysis of a phase 3 randomized clinical trial. J Diabetes Investig 9: 840-849.

23. Ma C, Sheng J, Liu Z, Guo M (2017) Excretion rates of 1,5-anhydro-D-glucitol, uric acid and microalbuminuria as glycemic control indexes in patients with type 2 diabetes. Sci Rep 7: 44291.

24. Chan CL, Pyle L, Kelsey MM, Newnes L, Baumgartner A, et al. (2016) Alternate glycemic markers reflect glycemic variability in continuous glucose monitoring in youth with prediabetes and type 2 diabetes. Pediatr Diabetes 18 : 629-636.

25. Li FF, Jiang L, Fu L, Zhu HH, Zhou P, et al. (2017) Exenatide add-on to continuous subcutaneous insulin infusion therapy reduces bolus insulin doses in patients with type 2 diabetes: a randomized, controlled, open-label trial. Diabetes Ther 8: 177-187.

26. Ke W, Liu L, Liu J, Chen A, Deng W, et al. (2016) Effects of liraglutide combined with short-term continuous subcutaneous insulin infusion on glycemic control and beta cell function in patients with newly diagnosed type 2 diabetes mellitus: a pilot study. J Diabetes Res 2016: 
6839735.

27. Tangvarasittichai S (2015) Oxidative stress, insulin resistance, dyslipidemia and type 2 diabetes mellitus. World $J$ Diabetes 6: 456-480.

28. Garcia-Bailo B, El-Sohemy A, Haddad PS, Arora P, Benzaied F, et al. (2011) Vitamins D, C, and E in the prevention of type 2 diabetes mellitus: modulation of inflammation and oxidative stress. Biologics 5: 7-19.

29. Hendarto $H$, Inoguchi $T$, Maeda $Y$, Ikeda $N$, Zheng J, et al. (2012) GLP-1 analog liraglutide protects against oxidative stress and albuminuria in streptozotocin-induced diabetic rats via protein kinase A-mediated inhibition of renal NAD(P)H oxidases. Metabolism 61: 1422-1434.

30. Gao H, Zeng Z, Zhang H, Zhou X, Guan L, et al. (2015) The glucagon-like peptide-1 analogue liraglutide inhibits oxidative stress and inflammatory response in the liver of rats with diet-induced non-alcoholic fatty liver disease. Biol Pharm Bull 38: 694-702.

31. Tatsch E, De Carvalho JA, Hausen BS, Bollick YS, Torbitz VD, et al. (2015) Oxidative DNA damage is associated with inflammatory response, insulin resistance and microvascular complications in type 2 diabetes. Mutat Res 782: 17-22.
32. Maschirow L, Khalaf K, Al-Aubaidy HA, Jelinek HF (2015) Inflammation, coagulation, endothelial dysfunction and oxidative stress in prediabetes-Biomarkers as a possible tool for early disease detection for rural screening. Clin Biochem 48: 581-585.

33. Nishikawa $T$, Sasahara $T$, Kiritoshi S, Sonoda K, Senokuchi T, et al. (2003) Evaluation of urinary 8hydroxydeoxy-guanosine as a novel biomarker of macrovascular complications in type 2 diabetes. Diabetes Care 26: $1507-1512$.

34. Broedbaek K, Weimann A, Stovgaard ES, Poulsen HE (2011) Urinary 8-oxo-7,8-dihydro-2'-deoxyguanosine as a biomarker in type 2 diabetes. Free Radic Biol Med 51: 1473-1479.

35. Toyokuni S, Yamada S, Kashima M, Ihara Y, Yamada Y, et al. (2000) Serum 4-hydroxy-2-nonenal-modified albumin is elevated in patients with type 2 diabetes mellitus. Antioxid Redox Signal 2: 681-685.

36. Elrayess MA, Almuraikhy S, Kafienah W, Al-Menhali A, Al-Khelaifi F, et al. (2017) 4-hydroxynonenal causes impairment of human subcutaneous adipogenesis and induction of adipocyte insulin resistance. Free Radic Biol Med 104: 129-137. 